\title{
Efficacy and safety of a combination of azithromycin and chloroquine for the treatment of uncomplicated Plasmodium falciparum malaria in two multi-country randomised clinical trials in African adults
}

Issaka Sagara ${ }^{1}$, Abraham R Oduro ${ }^{2}$, Modest Mulenga $^{3}$, Yemou Dieng ${ }^{4}$, Bernhards Ogutu ${ }^{5}$, Alfred B Tiono ${ }^{6}$,

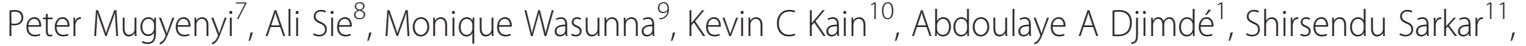
Richa Chandra ${ }^{12}$, Jeffery Robbins ${ }^{13^{*}}$ and Michael W Dunne ${ }^{14}$

\begin{abstract}
Background: Given increasing rates of resistance to existing therapy, new options for treatment and prophylaxis of malaria are needed.

Methods: Two randomised, comparative, non-inferiority studies were conducted in Africa, one double-blinded and one open-label. Adults with fever, a positive peripheral blood smear, and a positive rapid diagnostic test for Plasmodium falciparum were randomised in both studies to either azithromycin (AZ) 1,000 mg plus chloroquine (CQ) 600-mg base (AZCQ 1,000 mg) once daily for three days or mefloquine hydrochloride (MQ) 1,250 mg (split dose). In the first study, an additional regimen of AZ $500 \mathrm{mg}$ plus CQ 600-mg base (AZCQ $500 \mathrm{mg}$ ) once daily for three days was included. All study participants were hospitalised until three consecutive daily blood smears were negative for asexual $P$. falciparum parasitaemia. Study participants were evaluated weekly for 42 days, with Day 28 polymerase chain reaction (PCR)-corrected parasitological clearance rate as primary endpoint.

Results: A total of 467 subjects were randomised in the two studies. At 28 days' follow-up, PCR-corrected parasitological clearance rates in the per protocol population in the first study were 101/103 (98\%) with AZCQ $1,000 \mathrm{mg}$ compared with 102/103 (99\%) with MQ (95\% confidence interval [Cl]: -5.2, 3.3). The AZCQ 500-mg regimen was stopped during an interim study review (six [86\%] clearance of seven evaluable; two lost to follow-up). In the second study, clearance rates were similar: AZCQ 1,000 mg 107/107 (100\%) vs MQ 111/112 (99\%; 95\% Cl: -1.8, 3.6). Among the participating countries, in vitro CQ resistance based on pfcrt mutation frequency in the baseline isolates across both studies ranged from 20.8\% (Zambia) to 96.1\% (Uganda). Serious adverse events (AEs; all causality) were observed more frequently with MQ compared with AZCQ (four vs one, respectively), though discontinuations for AEs were similar (four vs three, respectively). Common AEs in the AZ-containing arms included pruritus, vomiting, dizziness, and headache.

(Continued on next page)
\end{abstract}

\footnotetext{
*Correspondence: jeffery.robbins@pfizer.com

${ }^{13}$ Global Research and Development, Pfizer Inc, 445 Eastern Point Road,

Groton, CT 06340, USA

Full list of author information is available at the end of the article
} 
(Continued from previous page)

Conclusions: Among adults with symptomatic uncomplicated falciparum malaria in Africa, the combination of AZ 1,000 mg and CQ 600-mg base once daily for three days resulted in Day 28 PCR-corrected parasitological clearance rates of $\geq 98 \%$ and was non-inferior to treatment with $M Q$. AZCQ was well tolerated.

Trial registration: ClinicalTrials.gov identifiers NCT00082576 and NCT00367653

Keywords: Azithromycin, Chloroquine, Falciparum malaria, Combination treatment

\section{Background}

Malaria remains one of the most common causes of morbidity and mortality in Africa. In 2011, >80\% of the population in most countries in Africa were at high risk for malaria [1]. The use of pesticides and the broad availability of chloroquine (CQ) effectively contained malaria on the African continent through the 1970s, until subsequent development of resistance by the Plasmodium species led to increased morbidity and mortality [2].

Management of malaria in Africa requires a variety of preventive and treatment initiatives, each of which targets different patient subpopulations. As a consequence, these initiatives demand medical therapy that, individually, vary in the requirements for safety, efficacy, and tolerability and, collectively, must integrate within a larger public health strategy. Increasing degrees of parasite resistance to mainstream drug interventions have further limited the choice of therapy available for malaria [3]. For example, there is currently no generally acceptable alternative for sulphadoxine/pyrimethamine in the intermittent preventive therapy in pregnancy programme. New therapy is needed and alternative options must be explored for their potential utility.

The anti-malarial properties of azithromycin (AZ) have been documented in vitro and in animal experiments, as well as in treatment and prevention clinical trials of both Plasmodium falciparum and Plasmodium vivax [4-8]. Given as monotherapy, AZ did not meet clinical standards of efficacy for treatment of falciparum malaria. However, further in vitro work suggested the possibility of synergy between AZ and CQ [4], which was subsequently supported by a small clinical trial in India [6].

Given the need for a new anti-malarial therapy and the scarcity of new potential agents, and the promising early results of a pilot clinical study [6], as well as the well-established safety profiles of both agents, there was a need to conduct a study to compare combined $\mathrm{AZ}$ and $\mathrm{CQ}$ with mefloquine hydrochloride (MQ). MQ was used as the comparator based on the recommendation of the US Food and Drug Administration (FDA). The results of two trials of the combination of $A Z$ and $C Q$ in the treatment of symptomatic uncomplicated P. falciparum malaria in adults in Africa are reported here.

\section{Methods}

\section{Study design}

The two phase II/III multi-centre, parallel, randomised, controlled, comparative, non-inferiority studies reported here were performed sequentially. Study number A0661134 (referred to hereafter as study 1134; ClinicalTrials.gov identifier NCT00082576; double-blind phase II/III) was conducted between June 2004 and May 2006, and study number A0661155 (referred to hereafter as study 1155; ClinicalTrials.gov identifier NCT00367653; open-label phase III) was conducted between November 2006 and September 2007. Both studies were conducted in African countries; study 1134 in Ghana, Mali, Zambia, Kenya, and Uganda and study 1155 in Ghana, Mali, Zambia, Kenya, Burkina Faso, and Senegal. In both trials, the comparator agent was MQ and both studies were identical in conduct and analysis with two exceptions. Study 1134 was conducted with blinded therapy and initially included a third arm (AZ $500 \mathrm{mg}$ plus a CQ 600-mg base [AZCQ $500 \mathrm{mg}$ ] once daily for three days), whereas in study 1155 medication was provided unblinded with the same two treatment regimens as in study 1134. As a consequence, the methods described below relate to study conduct and analysis for both trials with exceptions specifically noted where relevant.

\section{Study population}

Eligible subjects were $\geq 18$ years of age with symptomatic uncomplicated malaria as demonstrated by blood smears positive for $P$. falciparum asexual parasitaemia between 1,000 and 100,000 parasites/ $\mu \mathrm{L}$ and fever, documented or by history, within the prior 24 hours. Subjects also were required to have a serum glucose $\geq 60 \mathrm{mg} / \mathrm{dL}$ and a rapid diagnostic test positive for $P$. falciparum. Women of childbearing potential were required to have a negative urine human chorionic gonadotropin test before study entry, and enrolled subjects were to use adequate contraception during the entire study. Subjects were excluded for any of the following reasons: clinical or laboratory evidence of severe or complicated malaria; the presence of non-falciparum species on microscopy; pregnancy or breast-feeding; history of allergy or 
hypersensitivity to $\mathrm{AZ}, \mathrm{CQ}, \mathrm{MQ}$, or related compounds; history of epilepsy or psoriasis; treatment with any antimalarial drug or with any antibacterial with known antimalarial activity within two weeks before enrolment into the study; laboratory evidence of abnormal renal or liver function; any major psychiatric disorders; inability to swallow oral medication; treatment with other investigational drugs 30 days prior to enrolment; alcohol and/or any other drug abuse; requirement to use medication during the study that might have interfered with the evaluation of the study drug; other medical conditions that would have interfered with the evaluation of the therapeutic response or safety of the study drug; and inability to comprehend and/or unwillingness to follow the study protocol; or, prior participation in this study. Finally, subjects were excluded if they had not lived continuously in a malaria-endemic area for at least the previous year.

Subjects were to be withdrawn from the study and placed on alternative therapy for any of the following reasons: impaired consciousness, respiratory distress, seizures, hypoglycaemia, gross haematuria, increase in parasitaemia to $>100,000$ parasites $/ \mu \mathrm{L} 48$ hours after the first treatment dose, failure of parasitaemia to decrease to $25 \%$ of the baseline value on Day 2 and investigator opinion that other anti-malarial therapy was indicated or treatment failure.

\section{Drug treatment}

Study drug was administered as blinded therapy with matching placebo (in study 1134) or unblinded (in study $1155)$. In study 1134 , eligible subjects were initially randomised to oral treatment for three days in one of three active treatment arms: AZ 1,000 mg plus a CQ 600-mg base (AZCQ 1,000 mg) once daily for three days, AZ $500 \mathrm{mg}$ plus a CQ 600-mg base (AZCQ $500 \mathrm{mg}$ ) once daily for three days, or MQ (salt equivalent to $28-\mathrm{mg}$ free base) 1,250 $\mathrm{mg}$ (initial 750-mg dose followed by $500 \mathrm{mg}$ on Day 0). In study 1155, subjects were randomised to either AZCQ 1,000 mg once daily for three days or MQ (salt equivalent to $28-\mathrm{mg}$ free base) $1,250 \mathrm{mg}$ (initial 750-mg dose followed by $500 \mathrm{mg}$ on Day 0 ). MQ was used as the comparator based on the recommendation of the FDA, as this was the only FDA-approved oral anti-malaria agent at the time. MQ was expected to have high efficacy rates in all of the chosen study locations at the time of the study conduct.

\section{Parasite identification}

A blood dipstick-based test (Binax NOW $^{\circledR}$ ICT, Scarborough, ME, USA) was used for rapid diagnostic testing of P. falciparum. Giemsa-stained blood smears were read and interpreted by experienced microscopists who were blinded to all clinical information, including treatment allocation and the readings by the other microscopists. Three slides were prepared for each subject; two of these were read locally, whereas the third slide was maintained for possible third party review in case of any discordance $\geq 50 \%$ between the first two readers. Microscopy results at the site guided subject management; any subject with persistent or recurrent parasitaemia during the follow-up period was treated with antimalarial drugs according to local treatment guidelines and withdrawn from the study after documentation of clearance of parasitaemia.

For subjects who developed asexual parasitaemia after a period of clearance, paired blood blots from baseline and the time of recurrence were analysed to distinguish recrudescence from re-infection. Genotyping was performed by nested polymerase chain reaction (PCR) at two different laboratories, the Naval Medical Research Unit 2 in Jakarta, Indonesia (study 1134 only) and the Malaria Research and Training Centre in Bamako, Mali (both studies). In both studies, merozoite surface protein (MSP)-1, MSP-2, and the microsatellite CA1 gene loci $\mathrm{Su}$ were amplified at the Malaria Research and Training Centre. Recrudescence was defined as the reappearance of asexual blood-stage parasites of the same genotype as Day 0 parasites, whereas re-infection was defined as infection by a different genotype. Subjects were censored at the time of re-infection.

Paired blood specimens, collected before treatment and at the time of recurrent asexual parasitaemia (treatment failure), were evaluated for mutations in the $P$. falciparum CQ resistance transporter gene (pfcrt). For study 1134, molecular detection of the K76T mutation associated with CQ resistance was detected by means of a standardised real-time PCR-based diagnostic assay $[9,10]$. In addition, specimens collected on Day 0 from subjects who responded to treatment also were analysed for $p f c r t$ gene mutations. Based on differential melting-curve analysis, isolates were identified as CQ-sensitive isolates with a CVMNK haplotype or CQ-resistant isolates carrying a CVIET, SVMNT, CVMNT, or CVMET haplotype. For study 1155, nested PCR methods were performed for $p f c r t$ (K76T) and P. falciparum multi-drug resistance protein-1 (pfmdr1; N86Y) with the specific primer pairs followed by restriction fragment length polymorphism [11].

\section{Study assessments}

All subjects were hospitalised and monitored until three consecutive blood smears were negative for asexual $P$. falciparum parasitaemia and the investigator deemed discharge from the hospital appropriate. Peripheral blood smears for parasite counts were obtained at eighthour intervals until clearance was demonstrated, then on Day 7 and weekly thereafter through Day 42 to 
monitor recrudescence. Vital signs, clinical signs and symptoms, adverse events (AEs), and concomitant medications were assessed for all subjects on each day of treatment (Days 0, 1, and 2) and at each post-therapy visit (Days 7, 14, 21, 28, 35, and 42). Haematology and serum chemistry laboratory tests were performed at Baseline and Day 3 and at subsequent visits if clinically indicated. Haematology tests included red blood count, white blood count with differential, haemoglobin, haematocrit, and platelets. Serum chemistry tests included electrolytes, urea nitrogen, creatinine, aspartate aminotransferase, alanine aminotransferase, and alkaline phosphatase.

\section{Efficacy}

The primary endpoint was the asexual $P$. falciparum parasite clearance rate, adjusted for molecular testing (PCRcorrected) to differentiate recrudescence (true failures) from re-infection. Efficacy was assessed at Days 28 (primary endpoint) and 42 in the per protocol (PP) population. Treated subjects were excluded from the PP population if they did not meet the disease definition, did not receive all three days of study medication (unless designated a treatment failure, defined as the development of signs of severe malaria in the presence of parasitaemia), or did not have a blood smear at the specified time point (unless due to recurrent parasitaemia before that time point). Subjects were assigned a response of eradicated if parasitaemia cleared within seven days after initiation of treatment and did not recur through the time point of interest. Failure was defined as not achieving clearance of asexual $P$. falciparum parasitaemia within seven days or, if after achieving clearance, PCR-corrected parasitaemia recurred.

Secondary efficacy analyses included time required for asexual parasite clearance, fever clearance time, and assessment of the percentage of subjects with early and late treatment failures as defined by the World Health Organization [12].

\section{Safety}

All subjects who received at least one dose of study medication were evaluated for safety. Subjects were monitored closely for clinical evidence of illness progression. All subjects with persistent or recurrent parasitaemia received therapy consistent with the local standard of care and were monitored until parasite clearance was documented. Other safety evaluations included AE and vital sign monitoring throughout the study, haematology and serum chemistry laboratory evaluations, and physical examinations.

\section{Statistical analyses}

The primary efficacy analysis compared the PCR-corrected asexual $P$. falciparum parasite clearance rates in the AZCQ $1,000 \mathrm{mg}$ and MQ treatment arms at Day 28 in the PP population. A two-sided confidence interval (CI), using the appropriate confidence level, was constructed for the difference between treatment groups in asexual parasite clearance rates using normal approximation to the binomial, and also for within group rates. A CI was computed for the clearance rate within a treatment group using exact methods when no failures were observed. The confidence level in the final analysis for study 1134 required a small adjustment upward from 95 to $95.04 \%$ to prevent the overall false-positive rate from exceeding 0.05 , due to the planned interim analysis of the primary efficacy outcome. There was no adjustment for centres in the analyses. Non-inferiority was to be concluded if the lower boundary of the $\mathrm{CI}$ for the difference in parasite clearance rates (AZCQ 1,000 mg MQ) was $-10 \%$ or more. A non-inferiority margin of $10 \%$ was based on regulatory guidance available at the time of study conception. Clearance time comparisons up to Day 7 were generated using Kaplan-Meier estimates. Parasite clearance time was defined as the time in days from Baseline to the first of the three consecutive zero parasite counts. Fever clearance time was defined as the time in days from Baseline to the first of the two consecutive time points without evidence of elevated temperature.

Total sample size was determined based on the following assumptions: $80 \%$ power to show non-inferiority of the AZCQ 1,000-mg arm relative to the MQ arm, 85\% of randomised subjects satisfying criteria for inclusion in the PP analysis at the Day 28 visit, and expected parasite clearance rates of $95 \%$ in the AZCQ 1,000-mg arm and 95\% in the MQ arm.

These studies were conducted in compliance with the Declaration of Helsinki, institutional review boards, informed consent regulation, and the International Conference on Harmonisation Good Clinical Practice guidelines. All local regulatory requirements were followed. The clinical protocol was conducted in accordance with FDA regulations. Pfizer Inc conducted the clinical monitoring of the study.

\section{Results}

A total of 467 subjects were randomised to study medication (Figure 1). The demographic and baseline characteristics of subjects were similar among treatment allocations (Table 1). Similar numbers of subjects on each regimen completed therapy.

The AZCQ 500-mg regimen was stopped early in study 1134 on the recommendation of the data safety monitoring board after a review of data from studies conducted in South America and India demonstrated a dose response with lower efficacy rates for this regimen compared with AZCQ 1,000 mg. At that point, a total of nine subjects had been randomised to the AZCQ 500-mg regimen, of which six of seven subjects in the PP population had responded to therapy through Day 28 (Figure 1). 


\section{A Study 1134}

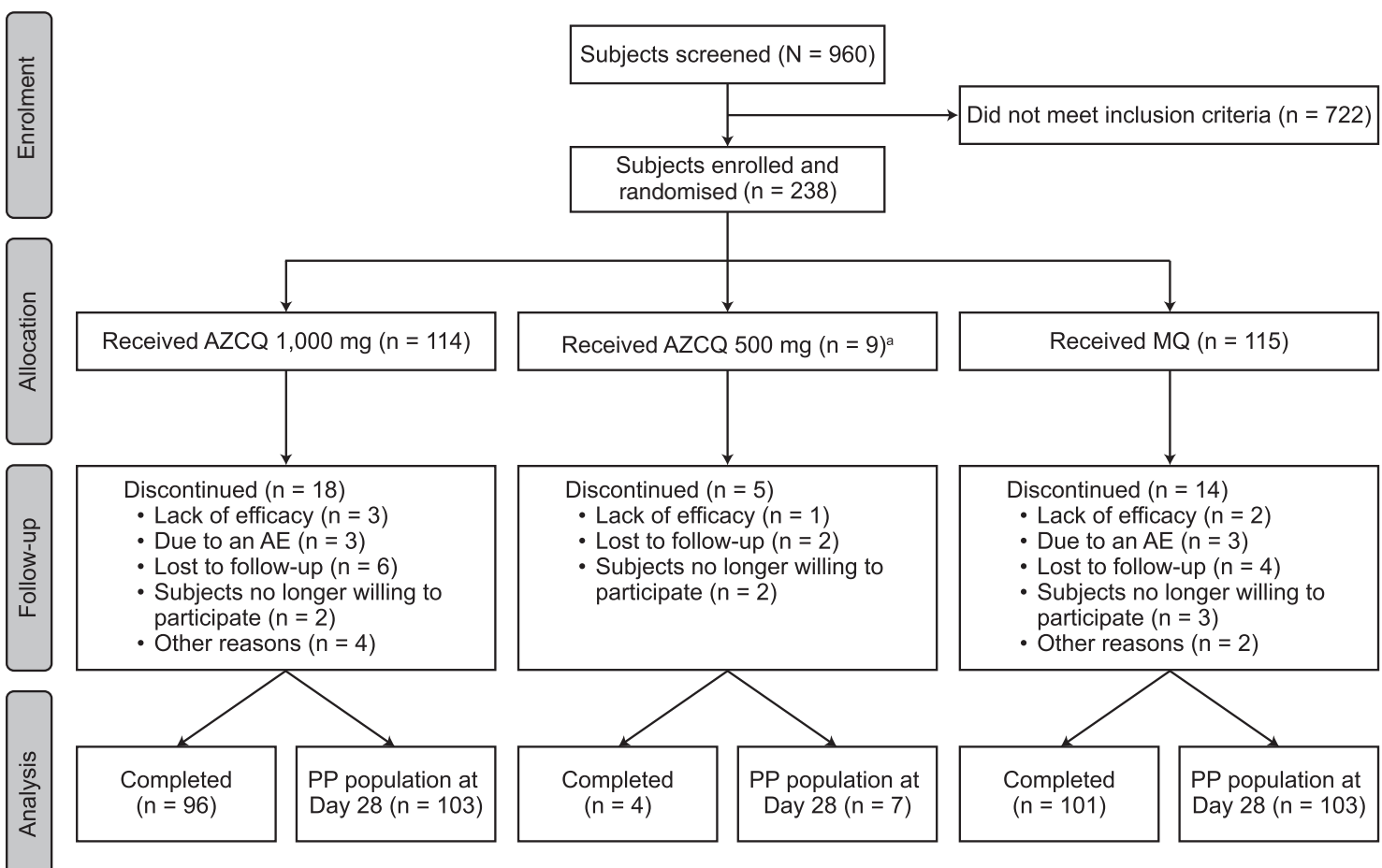

\section{B Study 1155}
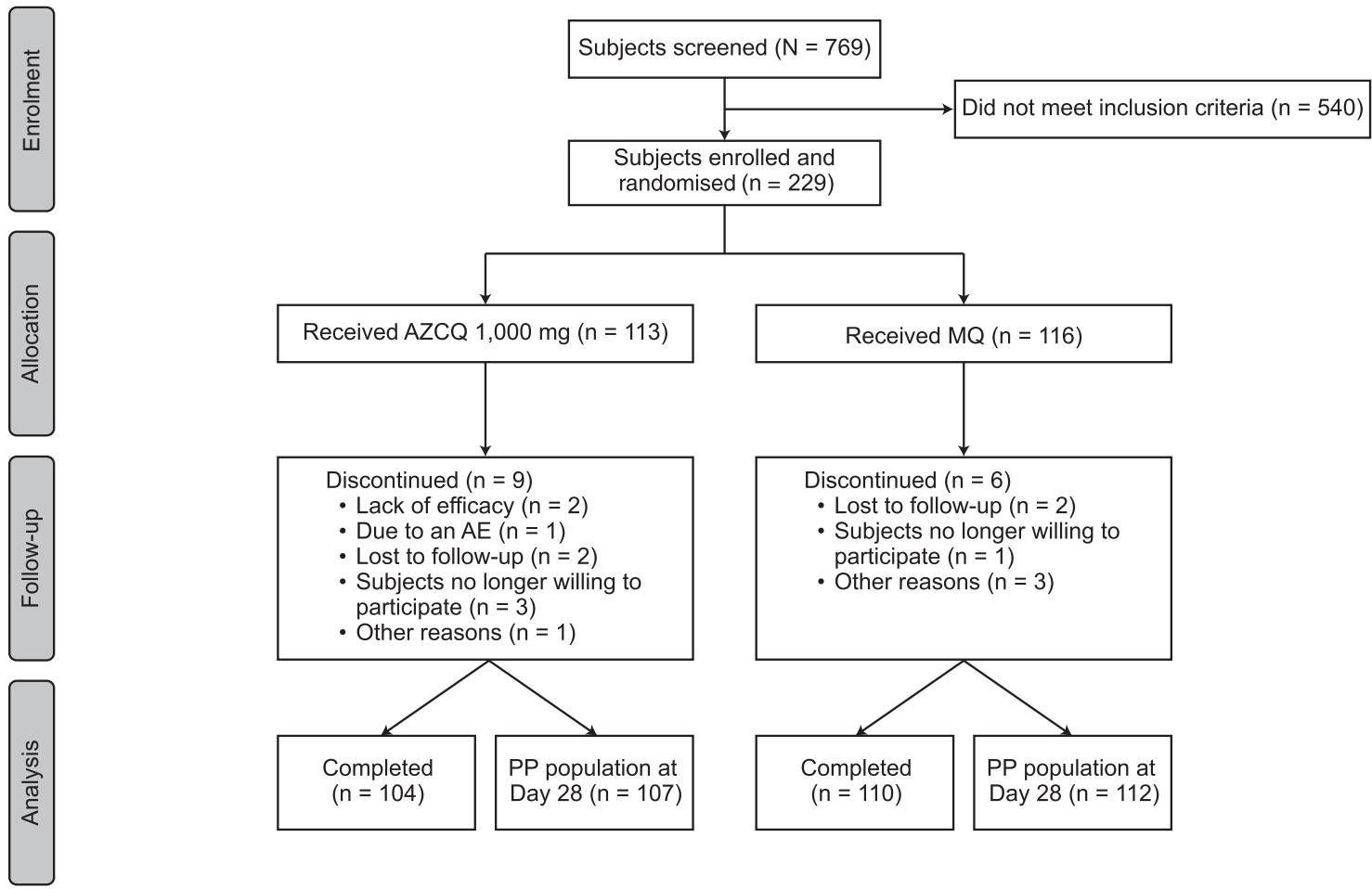

Figure 1 (See legend on next page.) 
(See figure on previous page.)

Figure 1 Subject disposition in study 1134 (A) and study 1155 (B). ${ }^{\text {TT }}$ The AZCQ 500-mg regimen was not included in the analysis, as treatment was stopped early on the recommendation of the data safety monitoring board after a review of data from studies conducted in South America and India demonstrated a dose response with lower efficacy rates for this regimen compared with AZCQ 1,000 mg. AE, adverse event; AZCQ 1,000 mg, azithromycin 1,000 mg plus chloroquine 600-mg base; AZCQ 500 mg, azithromycin 500 mg plus chloroquine 600-mg base; MQ, mefloquine hydrochloride; PP, per protocol.

All subjects with persistent or recurrent parasitaemia during the follow-up period were to receive rescue therapy with an anti-malarial regimen that was consistent with the local standard of care. Ten and five subjects treated with AZCQ and five and three patients treated with MQ in studies 1134 and 1155 , respectively, received rescue medication. The most common rescue mediation used was quinine in both treatment groups.

Among study 1134 PP population subjects at Day 28, those randomised to AZCQ 1,000 $\mathrm{mg}$ had $98.1 \%$ parasite clearance $(94.9,10095.04 \% \mathrm{CI} ; \mathrm{n}=101$ of 103) compared with $99.0 \%$ parasite clearance $(96.7,100$ 95.04\% CI; $\mathrm{n}=102$ of 103) in those who received MQ based on PCR-corrected results (Table 2; difference, $-0.97 \%$ [95.04\% CI: $-5.2,3.3]$ ). For corresponding PCRuncorrected results, those randomised to AZCQ 1,000 mg had $95.1 \%$ parasite clearance $(90.5,99.895 .04 \% \mathrm{CI} ; \mathrm{n}=98$ of 103) compared with $98.1 \%$ parasite clearance $(94.9,100$ $95.04 \% \mathrm{CI} ; \mathrm{n}=101$ of 103) in those who received MQ. At
Day 42, 99\% parasite clearance was observed in both groups (PCR-corrected; 96.6, $10095.04 \% \mathrm{CI} ; \mathrm{n}=100$ of 101 AZCQ; 96.6, 100 95.04\% CI; $\mathrm{n}=100$ of $101 \mathrm{MQ}$; difference, 0\% [95.04\% CI: $-3.74,3.74]$ ).

The rate of gametocyte clearance was similar in both AZCQ and MQ groups in both studies and was >95\% from Day 7 onwards.

The median time to clearance of asexual $P$. falciparum parasitaemia was 48 hours for those given AZCQ $1,000 \mathrm{mg}$ and 36 hours for those given MQ $(\mathrm{p}=0.0226)$. Median time to resolution of fever was observed to be $<0.5$ days after starting therapy for subjects randomised to AZCQ 1,000 mg and 0.5 days for those given MQ.

Similar findings were observed in study 1155 , whereas subjects in the PP population randomised to AZCQ 1,000 $\mathrm{mg}$ had 100\% parasite clearance $(96.6,100$ 95\% CI; $\mathrm{n}=107$ of 107) compared with $99.1 \%$ (96.9, $10095 \% \mathrm{CI}$; $\mathrm{n}=111$ of 112) in those randomised to MQ (difference,

Table 1 Subject demographics

\begin{tabular}{|c|c|c|c|c|}
\hline & \multicolumn{2}{|c|}{ Study 1134} & \multicolumn{2}{|c|}{ Study 1155} \\
\hline & $\begin{array}{c}\text { AZCQ } \\
1,000 \mathrm{mg} \\
(\mathrm{N}=114)\end{array}$ & $\begin{array}{c}\mathrm{MQ} \\
1,250 \mathrm{mg} \\
(\mathrm{N}=115)\end{array}$ & $\begin{array}{c}\text { AZCQ } \\
1,000 \mathrm{mg} \\
(\mathrm{N}=113)\end{array}$ & $\begin{array}{c}M Q \\
1,250 \mathrm{mg} \\
(\mathrm{N}=116)\end{array}$ \\
\hline \multicolumn{5}{|l|}{ Sex, n } \\
\hline Male & 66 & 61 & 65 & 63 \\
\hline Female & 48 & 54 & 48 & 53 \\
\hline \multicolumn{5}{|l|}{ Age group, years, $n$} \\
\hline $18-44$ & 106 & 101 & $100^{\mathrm{a}}$ & 99 \\
\hline $45-64$ & 8 & 13 & 13 & 15 \\
\hline$\geq 65$ & 0 & 1 & 0 & 2 \\
\hline Mean (SD) & $29.4(9.6)$ & $30.2(11.0)$ & $30.2(11.0)$ & $31.2(12.4)$ \\
\hline \multicolumn{5}{|l|}{ Weight, kg } \\
\hline Mean (SD) & $61.4(10.8)$ & $60.4(11.0)$ & $60.0(9.9)$ & $59.4(10.1)$ \\
\hline Range & $40.0-105.0$ & $39.0-107.0$ & $40.0-83.6$ & $39.0-94.0$ \\
\hline Baseline parasite count $/ \mu \mathrm{L}$, mean $\pm \mathrm{SD}$ & $20,889 \pm 24,176$ & $20,146 \pm 27,564$ & $16,686 \pm 22,863$ & $17,651 \pm 22,435$ \\
\hline Range & $160-111,040$ & $1,040-164,600$ & $120-93,440$ & $1,000-97,234$ \\
\hline \multicolumn{5}{|c|}{ Number of episodes of malaria in previous 2 years } \\
\hline 0 & 61 & 66 & 69 & 67 \\
\hline 1 & 20 & 18 & 9 & 14 \\
\hline 2 & 15 & 9 & 16 & 18 \\
\hline$\geq 3$ & 17 & 22 & 19 & 17 \\
\hline
\end{tabular}

${ }^{\mathrm{a}}$ One subject $<18$ years of age.

AZCQ 1,000 mg, azithromycin 1,000 mg plus chloroquine 600-mg base; MQ, mefloquine hydrochloride; SD, standard deviation. 
Table 2 Summary of efficacy outcomes at Day 28 in the parasitological per protocol population

\begin{tabular}{|c|c|c|c|c|}
\hline & \multicolumn{2}{|c|}{ Study 1134} & \multicolumn{2}{|c|}{ Study 1155} \\
\hline & $\begin{array}{c}\text { AZCQ } \\
1,000 \mathrm{mg}\end{array}$ & $\begin{array}{c}\mathrm{MQ} \\
1,250 \mathrm{mg}\end{array}$ & $\begin{array}{c}\text { AZCQ } \\
1,000 \mathrm{mg}\end{array}$ & $\begin{array}{c}\mathrm{MQ} \\
1,250 \mathrm{mg}\end{array}$ \\
\hline All treated, $\mathrm{N}$ & 114 & 115 & 113 & 116 \\
\hline PP population, $n$ & 103 & 103 & 107 & 112 \\
\hline \multicolumn{5}{|l|}{ PCR-corrected } \\
\hline $\begin{array}{l}\text { Eradicated, n (\%) } \\
(95 \% \mathrm{Cl})\end{array}$ & $\begin{array}{c}101(98.1) \\
(94.9,100)^{\mathrm{a}}\end{array}$ & $\begin{array}{l}102(99.0) \\
(96.7,100)^{\mathrm{a}}\end{array}$ & $\begin{array}{l}107(100.0) \\
(96.6,100)\end{array}$ & $\begin{array}{l}111(99.1) \\
(96.9,100)\end{array}$ \\
\hline $\begin{array}{l}\text { Difference, \% } \\
(95 \% \text { Cl) }\end{array}$ & \multicolumn{2}{|c|}{$-0.97(-5.23,3.29)^{a}$} & \multicolumn{2}{|c|}{$0.89(-1.77,3.56)$} \\
\hline ETF, n (\%) & 0 & $1(1.0)$ & 0 & $1(0.9)$ \\
\hline LTF, n (\%) & $2(1.9)$ & 0 & 0 & 0 \\
\hline LPF, n (\%) & 2 & 0 & 0 & 0 \\
\hline LCF, n (\%) & 0 & 0 & 0 & 0 \\
\hline $\begin{array}{l}\text { Median fever } \\
\text { clearance time, days }\end{array}$ & $<0.5$ & 0.5 & 1.5 & 1.0 \\
\hline $\begin{array}{l}\text { Median parasite } \\
\text { clearance time, } \mathrm{h}\end{array}$ & 48 & 36 & 44 & 40 \\
\hline
\end{tabular}

${ }^{a} 95.04 \% \mathrm{Cl}$ (small adjustment to the $95 \% \mathrm{Cl}$ required in order to account for a planned interim look at the primary efficacy outcome).

AZCQ 1,000 mg, azithromycin 1,000 mg plus chloroquine $600-\mathrm{mg}$ base; $\mathrm{Cl}$, confidence interval; ETF, early treatment failure; $L C F$, late clinical failure; $\mathrm{LPF}$, late parasitological failure; LTF, late treatment failure; $M Q$, mefloquine hydrochloride; PCR, polymerase chain reaction; PP, per protocol.

0.89\% [95\% CI: $-1.8,3.6]$ ) at Day 28. For corresponding PCR-uncorrected results, subjects in both the AZCQ 1,000-mg (96.8, $10095 \% \mathrm{CI} ; \mathrm{n}=106$ of 107) and MQ (96.9, $10095 \% \mathrm{CI} ; \mathrm{n}=111$ of 112 ) groups had a $99.1 \%$ parasite clearance. At Day 42, PCR-corrected parasite clearance remained at $100 \%$ for the AZCQ group $(96.5,100$ $95 \% \mathrm{CI} ; \mathrm{n}=104$ of 104$)$ and $99.1 \%$ for the MQ group (96.9, $10095 \% \mathrm{CI} ; \mathrm{n}=110$ of 111 ; difference, $0.90 \%$ [95\% CI: $-1.8,3.6])$.

The median time to clearance of parasitaemia was 44 hours for subjects given AZCQ 1,000 $\mathrm{mg}$ and 40 hours for those given MQ. Median time to resolution of fever was observed to be 1.5 days after starting therapy for subjects randomised to AZCQ 1,000 $\mathrm{mg}$ and 1 day for those given $\mathrm{MQ}$, although the difference was not statistically significant.

Two late parasitological failures (LPFs) were observed in study 1134 for subjects in the AZCQ 1,000-mg treatment group (Table 2), whereas there were no LPFs in the MQ treatment group. No LPFs were observed in study 1155 in either treatment group.

Paired isolates from the two subjects who demonstrated a recrudescence on AZCQ 1,000 mg were sequenced to identify possible signature resistance mutations. No such mutations were identified [13]. CQ-resistance-associated mutation rates varied among the countries participating in these studies, from $96.1 \%$ in Uganda (study 1134) to $20.8 \%$ in Zambia (study 1155; Table 3). An analysis of isolates collected during study 1134 indicated that $13 \%(15 / 115)$ of CQ-resistant isolates carried the CVMNT pfcrt haplotype (Table 4).

Treatment-emergent AEs considered related to study medication were observed in $78.1 \%(89 / 114)$ of subjects randomised to AZCQ 1,000 $\mathrm{mg}$ and in 61.7\% (71/115) of those randomised to MQ in study 1134, and in $70.8 \%$ $(80 / 113)$ and $62.1 \%(72 / 116)$ of subjects, respectively, in study 1155 (Table 5). Pruritus was seen more frequently with AZCQ 1,000 mg, whereas dizziness was seen more often with MQ (Table 5). Overall, three (1.3\%) subjects given AZCQ 1,000 mg discontinued therapy due to an $\mathrm{AE}$ related to study drug (vomiting, pruritus, vomiting/dizziness/tinnitus) compared with two (0.9\%) of those receiving MQ (fever/hypotension/haematuria, hypertension/vomiting). No clinically significant changes were observed in any laboratory values over the duration of the study and abnormal laboratory values did not result in any study discontinuations (see Additional files 1 and 2).

Table 3 Markers of chloroquine resistance in isolates obtained during the clinical trial

\begin{tabular}{|c|c|c|c|c|c|c|c|}
\hline \multirow{2}{*}{$\begin{array}{l}\text { Number of } \\
\text { subjects }\end{array}$} & \multirow[b]{2}{*}{ Tested } & \multicolumn{3}{|c|}{ Pfcrt } & \multicolumn{3}{|c|}{ Resistance mutations, \% } \\
\hline & & $\bar{K}$ & $T$ & KT & No band & $2004-2006^{a}$ & $2006-2007^{b}$ \\
\hline \multicolumn{8}{|l|}{ Ghana } \\
\hline Study 1134 & 27 & 11 & 16 & 0 & 0 & 59.3 & \\
\hline Study 1155 & 83 & 52 & 25 & 3 & 3 & & 33.7 \\
\hline \multicolumn{8}{|l|}{ Uganda } \\
\hline Study 1134 & 51 & 1 & 49 & 0 & 1 & 96.1 & \\
\hline \multicolumn{8}{|l|}{ Study 1155} \\
\hline \multicolumn{8}{|l|}{ Zambia } \\
\hline Study 1134 & 116 & 85 & 26 & 5 & 0 & 26.7 & \\
\hline Study 1155 & 77 & 61 & 13 & 3 & 0 & & 20.8 \\
\hline \multicolumn{8}{|l|}{ Mali } \\
\hline Study 1134 & 27 & 8 & 18 & 0 & 1 & 66.7 & \\
\hline Study 1155 & 29 & 5 & 20 & 4 & 0 & & 82.8 \\
\hline \multicolumn{8}{|l|}{ Burkina Faso } \\
\hline \multicolumn{8}{|l|}{ Study 1134} \\
\hline Study 1155 & 27 & 19 & 7 & 0 & 1 & & 25.9 \\
\hline \multicolumn{8}{|l|}{ Kenya } \\
\hline Study 1134 & 15 & 2 & 10 & 0 & 3 & 66.7 & \\
\hline Study 1155 & 7 & 1 & 5 & 0 & 1 & & 71.4 \\
\hline \multicolumn{8}{|l|}{ Senegal } \\
\hline \multicolumn{8}{|l|}{ Study 1134} \\
\hline Study 1155 & 6 & 3 & 2 & 1 & 0 & & 50.0 \\
\hline
\end{tabular}


Table 4 Number of subjects with pfcrt gene mutations in study 1134

\begin{tabular}{lcc}
\hline & $\begin{array}{c}\text { AZCQ } \\
\mathbf{1 , 0 0 0} \mathbf{~ m g} \\
(\mathbf{N}=\mathbf{1 1 4})\end{array}$ & $\begin{array}{c}\text { MQ } \\
\mathbf{1 , 2 5 0} \mathbf{~ m g} \\
\mathbf{( N = 1 1 4 )}\end{array}$ \\
\hline CQ-sensitive, $\mathrm{n}$ & 48 & 59 \\
CVMNK & 48 & 58 \\
SVMNK & 0 & 1 \\
CQ-resistant, $\mathrm{n}$ & 63 & 52 \\
CVMET & 0 & 1 \\
CVMNT & 9 & 6 \\
CVIET & 54 & 45 \\
Negative, $n$ & 3 & 2 \\
Other, $n$ & 0 & 1 \\
\hline AZCQ & &
\end{tabular}

AZCQ 1,000 mg, azithromycin 1,000 mg plus chloroquine $600-\mathrm{mg}$ base; $\mathrm{CQ}$ chloroquine; MQ, mefloquine hydrochloride; pfcrt, Plasmodium falciparum $\mathrm{CQ}$ resistance transporter gene.

Overall in both studies, treatment-related serious AEs were only observed in the MQ group (3/231 [1.3\%]; mental disorder, nephrotic syndrome/blood creatinine increased and intentional self-injury). A severe $\mathrm{AE}$ of vomiting was seen in one $(0.4 \%)$ subjects given AZCQ $1,000 \mathrm{mg}$, whereas six severe AEs were observed in five (2.2\%) subjects given MQ (vomiting, increased creatinine, psychosis, intentional self-injury, nephrosis, and dizziness). The majority of all other AEs with AZCQ $1,000 \mathrm{mg}$ and MQ were mild in severity.

\section{Discussion}

The fixed-dose combination of AZ 1,000 mg and a CQ $600-\mathrm{mg}$ base was found to be non-inferior to MQ in two randomised studies conducted in Africa in adults with symptomatic uncomplicated malaria due to
P. falciparum. In addition to similar clinical and parasitological outcomes, the AZCQ fixed-dose combination also achieved an overall efficacy rate of $99 \%$, which is consistent with other treatment modalities recommended in treatment guidelines for this subject population [12]. The mean time to resolution of parasitaemia was comparable in each regimen, as was the time to resolution of fever.

A pre-specified non-inferiority margin of $10 \%$ was based on regulatory guidance at the time of study conception. Although this margin has been used by other phase III trials as recent as 2011, many trials of late have switched to a more conservative margin of 5\% [14]. Given the level of parasite clearance achieved at Day 28 in studies 1134 and 1155 , lower limits of the CIs comparing the two treatments would indicate a difference relative to $\mathrm{MQ}$ of no worse than $5.23 \%$ and $1.77 \%$, respectively.

In Zambia, the rate of clinical resistance to $\mathrm{CQ}$ was $>70 \%$ in 2003 at the time of switching from CQ to artemether/lumefantrine. Based on this observation, it is clear that AZCQ displayed efficacy in areas with a high prevalence of CQ-resistant falciparum malaria.

Molecular marker resistance to CQ across these seven African countries between 2004 and 2007 ranged from approximately 21 to $96 \%$. There was a trend to lower rates of CQ resistance in the clinical trial sites in Ghana and Zambia over this time period, which may be related to anti-malarial policies in these countries $v s$ the other countries included in this study.

The pfcrt CQ-resistant haplotype most frequently described in Africa has been CVIET, which is thought to have possibly been introduced from southeast Asia through India [10]. As previously reported, the CVIET haplotype was again observed in this study. Notably, $13 \%$ of isolates in this study carried the CVMNT haplotype and were

Table 5 Treatment-emergent, treatment-related adverse events in $\geq 5 \%$ of subjects in any group in each study

\begin{tabular}{|c|c|c|c|c|}
\hline & \multicolumn{2}{|c|}{ Study 1134} & \multicolumn{2}{|c|}{ Study 1155} \\
\hline & $\begin{array}{c}\text { AZCQ } 1,000 \mathrm{mg} \\
(\mathrm{N}=114)\end{array}$ & $\begin{array}{c}M Q 1,250 \mathrm{mg} \\
(\mathrm{N}=115)\end{array}$ & $\begin{array}{c}\text { AZCQ } 1,000 \mathrm{mg} \\
(\mathrm{N}=113)\end{array}$ & $\begin{array}{l}\text { MQ 1,250 mg } \\
\quad(\mathrm{N}=116)\end{array}$ \\
\hline Any $A E, n(\%)$ & $89(78.1)$ & $71(61.7)$ & $80(70.8)$ & $72(62.1)$ \\
\hline Pruritus & $58(50.9)$ & $11(9.6)$ & $32(28.3)$ & $1(0.9)$ \\
\hline Dizziness & $11(9.6)$ & $26(22.6)$ & 18 (15.9) & 19 (16.4) \\
\hline Vomiting & $18(15.8)$ & $12(10.4)$ & $4(3.5)$ & $20(17.2)$ \\
\hline Headache & $15(13.2)$ & $11(9.6)$ & $20(17.7)$ & $25(21.6)$ \\
\hline Abdominal pain & $8(7.0)$ & $13(11.3)$ & $13(11.5)$ & $9(7.8)$ \\
\hline Nausea & $9(7.9)$ & $13(11.3)$ & $10(8.8)$ & $12(10.3)$ \\
\hline Asthenia & $6(5.3)$ & $11(9.6)$ & $9(8.0)$ & $3(2.6)$ \\
\hline Palpitations & $3(2.6)$ & $7(6.1)$ & - & - \\
\hline Diarrhoea & $6(5.3)$ & $5(4.3)$ & $11(9.7)$ & $4(3.4)$ \\
\hline Fatigue & - & - & $4(3.5)$ & $6(5.2)$ \\
\hline Pain & $2(1.8)$ & $2(1.7)$ & $6(5.3)$ & $1(0.9)$ \\
\hline
\end{tabular}

$\mathrm{AE}$, adverse event; $\mathrm{AZCQ} 1,000 \mathrm{mg}$, azithromycin 1,000 mg plus chloroquine 600-mg base; $\mathrm{MQ}$, mefloquine hydrochloride. 
recovered from Ghana and Zambia. Both the SVMNT and CVMNT CQ-resistant mutations have been previously observed in Africa $[15,16]$.

While no CQ monotherapy control arm was included in this trial, the historical rates of clinical response to $\mathrm{CQ}$ in areas with significant in vitro $\mathrm{CQ}$ resistance would be expected to be significantly lower than those observed on this combination therapy [17]. Whether the enhanced efficacy of the combination is a consequence of the direct effects of $A Z$ on the parasite or the effect of $\mathrm{AZ}$ on the resistance mechanisms mediating CQ resistance, or both, cannot be established from these data.

Moreover, anti-malarial immunity in malaria-endemic areas also can assist in parasite clearance and may play a role in the enhanced efficacy of the combination therapy, although innate immunity is equally likely to be effective in those subjects who received either AZ or CQ alone [18].

Discontinuations due to AEs were infrequent and numbers were similar in each regimen. Pruritus was seen more frequently with the AZCQ combination, a welldescribed effect associated with the use of CQ in subjects with melanoderma [19]. Central nervous system AEs were seen more frequently with $\mathrm{MQ}$, and again this is consistent with historical experience [20]. Gastrointestinal AEs occurred with both regimens to a similar degree.

For a regimen to be considered appropriate as a therapy for malaria, it first must demonstrate that it is safe and effective in the target subject population. Other considerations, however, should be addressed with regard to the AZCQ combination. CQ is no longer recommended for the treatment of malaria in Africa due to the generally high rates of background CQ resistance and subsequent poor clinical response to therapy throughout the continent. The position of the World Health Organization is that all therapy for the treatment of malaria should be combination therapy, primarily combinations that include an artemisinin derivative [21]. The combination therapy should include a partner drug with sufficient efficacy to protect the artemisinin from the development of resistance. Alternatively, new modelling suggests that the introduction of a variety of therapy may be more effective in managing the rate at which resistance appears [22].

A limitation of this study was the recruitment of adults with symptomatic uncomplicated malaria only and thus, other target populations, including pregnant women, should be studied, as it is known that pregnant women are more susceptible to malaria compared with non-pregnant women within the same age group [23]. The progressive decline in the efficacy of sulphadoxine/ pyrimethamine in Africa, concerns with artemisinin use in pregnancy [12], and the extensive safety database for both AZ and CQ suggest that studies to investigate the merits of this combination for the prevention of malaria during pregnancy would appear justified [24]. Other areas of study also may include use in the paediatric population and, given the activity of AZ in a variety of bacterial infections $[25,26]$, home-based management of fever.

A phase III trial (ClinicalTrials.gov identifier NCT01103063) that evaluated a blended tablet of AZ and CQ for intermittent preventive treatment of malaria in pregnancy was stopped early, as an interim analysis indicated it was unlikely to meet the primary endpoint (i.e., stopped for trial futility) defined as superiority of AZCQ over sulfadoxine/pyrimethamine in the proportion of patients meeting a sub-optimal pregnancy outcome (abortion [ $\leq 28$ weeks], stillbirth $[>28$ weeks], premature delivery $[<37$ weeks], low birth weight $[<2,500 \mathrm{~g}]$ live neonate, missing neonatal birth weight, or lost to follow-up). As the primary endpoint was analysed using the intent-to-treat population, lack of tolerability of the combination treatment may have resulted in a higher dropout rate leading to the inclusion of missing data as a failure in the interim analysis.

New therapy for the prevention and treatment of malaria in the context of a broader campaign of public health interventions is, therefore, needed to help limit the morbidity and mortality associated with this disease.

\section{Conclusions}

The results of this study indicate that among adults with symptomatic uncomplicated falciparum malaria in Africa, a fixed-dose combination of AZ 1,000 $\mathrm{mg}$ and a CQ 600-mg base once daily for three days resulted in Day 28 and Day 42 PCR-corrected parasitological clearance rates of $\geq 98 \%$. AZCQ was non-inferior to treatment with MQ and was well tolerated.

\section{Additional files}

Additional file 1: Table S1. Median change from Baseline to last observation in laboratory values in study 1134. Description: The data in the table provides details of laboratory values from study 1134.

Additional file 2: Table S2. Median change from Baseline to last observation in laboratory values in study 1155. Description: The data in the table provides details of laboratory values from study 1155.

\section{Abbreviations}

AE: Adverse event; AZ: Azithromycin; AZCQ: Azithromycin chloroquine combination; AZCQ 500 mg: Azithromycin 500 mg plus chloroquine 600-mg base; AZCQ 1,000 mg: Azithromycin 1,000 mg plus chloroquine 600-mg base; Cl: Confidence interval; CQ: Chloroquine; ETF: Early treatment failure; FDA: US Food and drug administration; LCF: Late clinical failure; LPF: Late parasitological failure; MQ: Mefloquine hydrochloride; MSP: Merozoite surface protein; PCR: Polymerase chain reaction; LTF: Late treatment failure; pfcrt: Plasmodium falciparum CQ resistance transporter gene; PP: Per protocol; SD: Standard deviation.

\section{Competing interests}

These studies were funded by Pfizer Inc. JR is a full-time employee of and owns shares in Pfizer Inc. MWD and RC were full-time employees of Pfizer Inc when these studies were conducted and own shares in Pfizer Inc. All other authors declare that they have no competing interests. 


\section{Authors' contributions}

JR contributed to data analysis and manuscript development. RC contributed to the development of the study protocol, leading the conduct of study number A0661155, interpretation of the data and writing the clinical study report. MM $A R O, I S, P M, M W, A S, A B T, B O, A A D$, and YD were principal study investigators and contributed in protocol development. KCK contributed to the analysis of resistance mutations. MWD was the development head for infectious diseases at Pfizer Inc when these studies were conducted and contributed to the development of the protocol, interpretation of the data, and preparation of the first draft of the manuscript. SS conducted the statistical analyses and contributed to writing the clinical study report. All authors reviewed and edited manuscript drafts and approved the final version of the manuscript.

\section{Acknowledgements}

The authors wish to thank all study volunteers, the study staff, and the heads/ management of all the study centres for the successful completion of this study. The authors also thank Jeanne Breen, MD (previously of Pfizer Inc), for help in the preparation of the manuscript, and Bob Burnside (Pfizer Inc) for assistance in statistical data management. Editorial assistance was provided by Aruna Seth, PhD, of Engage Scientific Solutions and funded by Pfizer Inc. This manuscript is published with permission from the Director, KEMRI. KCK was supported by the Canadian Institutes of Health Research (CIHR MOP-13721 and MOP-115160) and a Canada Research Chair in Molecular Parasitology.

\section{Author details}

'Malaria Research and Training Centre, University of Sciences, Techniques and Technologies, Bamako, Mali. ${ }^{2}$ Navrongo Health Research Centre, Navrongo, Ghana. ${ }^{3}$ Tropical Diseases Research Centre, Ndola, Zambia. ${ }^{4}$ Parasitology and Mycology Department, Faculty of Medicine, Pharmacy, and Odonto-Stomatology, University of Dakar, Dakar, Senegal. ${ }^{5}$ Walter Reed Project, Centre for Clinical Research, Kenya Medical Research Institute, Kisumu, Kenya. ${ }^{6}$ Centre National de Recherche et de Formation sur le

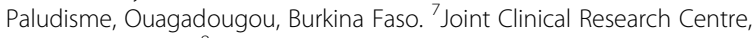
Kampala, Uganda. ${ }^{8}$ Centre de Recherche en Sante de Nouna, Nouna, Burkina Faso. ${ }^{9}$ Kenya Medical Research Institute, Nairobi, Kenya. ${ }^{10}$ Sandra A Rotman Laboratories, Sandra Rotman Center for Global Health, University Health Network, Toronto General Hospital, University of Toronto, Toronto, Ontario, Canada. ${ }^{11}$ Sciformix, Mumbai, India. ${ }^{12}$ Formerly of Pfizer Inc, New York, NY, USA. ${ }^{13}$ Global Research and Development, Pfizer Inc, 445 Eastern Point Road, Groton, CT 06340, USA. ${ }^{14}$ Durata Therapeutics, Inc., Morristown, NJ, USA.

Received: 7 April 2014 Accepted: 18 November 2014

Published: 25 November 2014

\section{References}

1. WHO: World Malaria Report. Geneva: World Health Organization; 2012 [http://www.who.int/malaria/publications/world_malaria_report_2012/en/]

2. Trape JF: The public health impact of chloroquine resistance in Africa. Am J Trop Med Hyg 2001, 64(Suppl 1-2):12-17.

3. Spalding MD, Eyase FL, Akala HM, Bedno SA, Prigge ST, Coldren RL, Moss WJ, Waters NC: Increased prevalence of the pfdhfr/phdhps quintuple mutant and rapid emergence of pfdhps resistance mutations at codons 581 and 613 in Kisumu, Kenya. Malar J 2010, 9:338.

4. Ohrt C, Willingmyre GD, Lee P, Knirsch C, Milhous W: Assessment of azithromycin in combination with other antimalarial drugs against Plasmodium falciparum in vitro. Antimicrob Agents Chemother 2002, 46:2518-2524.

5. Andersen SL, Oloo A, Gordon D, Ragama OB, Aleman GM, Berman JD, Tang DB, Dunne MW, Shanks GD: Successful double-blinded, randomized, placebo-controlled field trial of azithromycin and doxycycline as prophylaxis for malaria in western Kenya. Clin Infect Dis 1998, 26:146-150.

6. Dunne MW, Singh N, Shulka M: A double-blind, randomized study of azithromycin compared to chloroquine for the treatment of Plasmodium vivax malaria in India. Am J Trop Med Hyg 2005, 73:1108-1111.

7. Taylor WRJ, Richie TL, Fryauff DJ, Picarima H, Ohrt C, Tang D, Braitman D, Murphy GS, Widaja H, Tjitra E, Ganjar A, Jones TR, Basri H, Berman J: Malaria prophylaxis using azithromycin: a double-blind, placebo-controlled trial in Irian Jaya, Indonesia. Clin Infect Dis 1999, 28:74-81.

8. Dunne MW, Singh N, Shukla M, Valecha N, Bhattacharyya PC, Dev V, Patel K, Mohapatra MK, Lakhani J, Benner R, Lele C, Patki K: A multicenter study of azithromycin, alone and in combination with chloroquine, for the treatment of acute uncomplicated Plasmodium falciparum malaria in India. J Infect Dis 2005, 191:1582-1588.

9. Farcas GA, Soeller R, Zhong K, Zahirieh A, Kain KC: Real-time polymerase chain reaction assay for the rapid detection and characterization of chloroquine-resistant Plasmodium falciparum malaria in returned travelers. Clin Infect Dis 2006, 42:622-627.

10. Keen J, Farcas GA, Zhong K, Yohanna S, Dunne MW, Kain KC: Real-time PCR assay for rapid detection and analysis of PfCRT haplotypes of chloroquine-resistant Plasmodium falciparum isolates from India. I Clin Microbiol 2007, 45:2889-2893.

11. Djimde A, Doumbo OK, Cortese JF, Kayentao K, Doumbo S, Diourte Y, Coulibaly D, Dicko A, Su XZ, Nomura T, Fidock DA, Wellems TE, Plowe CV: A molecular marker for chloroquine-resistant falciparum malaria. $N$ Engl J Med 2001, 344:257-263.

12. Assessment and monitoring of antimalarial drug efficacy for the treatment of uncomplicated falciparum malaria. [http://whqlibdoc.who. int/hq/2003/WHO_HTM_RBM_2003.50.pdf]

13. Pereira MR, Henrich PP, Sidhu AS, Johnson D, Hardink J, Van Deusen J, Lin J, Gore K, O'Brien C, Wele M, Djimde A, Chandra R, Fidock DA: In vivo and in vitro antimalarial properties of azithromycin-chloroquine combinations that include the resistance reversal agent amlodipine. Antimicrob Agents Chemother 2011, 55:3115-3124

14. Poravuth $Y$, Socheat $D$, Rueangweerayut $R$, Uthaisin C, Pyae Phyo A, Valecha N, Rao BH, Tjitra E, Purnama A, Borghini-Fuhrer I, Duparc S, Shin CS, Fleckenstein L: Pyronaridine-artesunate versus chloroquine in patients with acute Plasmodium vivax malaria: a randomized, double-blind, non-inferiority trial. PLoS One 2011, 6:e14501.

15. Alifrangis M, Dalgaard MB, Lusingu JP, Vestergaard LS, Staalsoe T, Jensen ATR, Enevold A, Ronn AM, Khali IF, Warhurst DC, Lemnge MM, Theander TG, Bygbjerg IC: Occurrence of the Southeast Asian/South American SVMNT haplotype of the chloroquine-resistance transporter gene in Plasmodium falciparum in Tanzania. J Infect Dis 2006, 193:1738-1741.

16. Gbotosho GO, Folarin OA, Bustamante C, da Silva LHP, Mesquita E, Sowunmi A, Zalis MG, Oduola AMJ, Happi CT: Different patterns of pfcrt and pfmdr1 polymorphisms in $P$. falciparum isolates from Nigeria and Brazil: the potential role of antimalarial drug selection pressure. Am J Trop Med Hyg 2012, 86:211-213.

17. Attaran A, Barnes Kl, Curtis C, d'Alessandro U, Fanello Cl, Galinski MR, Kokwaro G, Looareesuwan S, Makanga M, Mutabingwa TK, Talisuna A, Trape JF, Watkins WM: WHO, the Global Fund, and medical malpractice in malaria treatment. Lancet 2004, 363:237-240.

18. Djimdé AA, Doumbo OK, Traore O, Guindo AB, Kayentao K, Diourte $Y$, Niare-Doumbo S, Coulibaly D, Kone AK, Cissoko Y, Tekete M, Fofana B, Dicko A, Diallo DA, Wellems TE, Kwiatkowski D, Plowe CV: Clearance of drug-resistant parasites as a model for protective immunity in Plasmodium falciparum malaria. Am J Trop Med Hyg 2003, 69:558-563.

19. Bussaratid V, Walsh DS, Wilairatana P, Krudsood S, Silachamroon U, Looareesuwan S: Frequency of pruritus in Plasmodium vivax malaria patients treated with chloroquine in Thailand. Trop Doct 2000, 30:211-214.

20. Baird JK: Effectiveness of antimalarial drugs. N Engl J Med 2005, 352:1565-1577.

21. Guidelines for the treatment of malaria [second ed]. [http://whqlibdoc. who.int/publications/2010/9789241547925_eng.pdf]

22. Boni MF, Smith DL, Laxminarayan R: Benefits of using multiple first-line therapies against malaria. Proc Natl Acad Sci U S A 2008, 105:14216-14221.

23. Desai M, ter Kuile FO, Nosten F, McGready R, Asamoa K, Brabin B, Newman RD: Epidemiology and burden of malaria in pregnancy. Lancet Infect Dis 2007, 7:93-104.

24. Chico RM, Pittrof R, Greenwood B, Chandramohan D: Azithromycinchloroquine and the intermittent preventive treatment of malaria in pregnancy. Malar J 2008, 7:255

25. Shanks GD, Smoak BL, Aleman GM, Oundo J, Waiyaki PG, Dunne MW, Petersen L: Single dose of azithromycin or three-day course of ciprofloxacin as therapy for epidemic dysentery in Kenya. Clin Infect Dis 1999, 29:942-943.

26. Pfizer Inc: Zithromax [prescribing information]. New York, NY: Pfizer Inc; 2013.

\section{doi:10.1186/1475-2875-13-458}

Cite this article as: Sagara et al.: Efficacy and safety of a combination of azithromycin and chloroquine for the treatment of uncomplicated Plasmodium falciparum malaria in two multi-country randomised clinical trials in African adults. Malaria Journal 2014 13:458. 